\title{
Myriad Obstetric Complications in Patients with Scarred Uterus: A Case Series
}

\author{
Sahana Rao ${ }^{1}$, Veena $\mathrm{Hadi}^{2}$, Ashalata Mallapur ${ }^{3}$, Chandalingappa Kuri ${ }^{4}$, Geetanjali Katageri ${ }^{5}$
}

\begin{abstract}
Obstetric practice in recent times has witnessed a steady increase in the rate of primary cesarean sections and thus, the consequences of the same. Managing previous cesarean cases in obstetrics has now become a routine, and the complications which we encounter in these patients. In this case series, we present five different presentations of previous cesareans and their outcomes. Treating the complications in these leads to a lot of diagnostic and management dilemmas as there is no definite prescribed protocol and individualized management strategies need to be adopted. Atypical clinical and radiological signs should be identified in such cases and should be managed in a tertiary care hospital for the best outcome of the patient.

Keywords: Cesarean section, Hysterectomy, Methotrexate, Placenta previa, Ultrasonography.

Journal of South Asian Federation of Obstetrics and Gynaecology (2020): 10.5005/jp-journals-10006-1843
\end{abstract}

\section{INTRODUCTION}

The increase in the rate of primary cesarean sections and then repeat cesarean sections in current obstetric practice has led to the emergence and increasing incidence of a variety of new obstetric problems, especially the scar site problems. Managing such cases poses new challenges in day-to-day practice. Possible rupture of the uterus during labor is the most widely discussed complication with available guidelines on trial of labor after cesarean and vaginal birth after cesarean. ${ }^{1}$ It is no longer true that the management of a case of previous cesarean is limited to only possible rupture during labor as is the standard teaching. The varied presentations and complications arising in these patients could pave a way for new obstetric chapters in the near future. Here we present a case series of varied complications in women with previous cesarean deliveries, and their outcomes.

\section{Case 1: Cesarean Scar Pregnancy Presenting as Incomplete Abortion}

Thirty-six-year-old Mrs. A presented to our institute with incomplete abortion with complaints of heavy vaginal bleeding. She was a third gravida with previous two-term cesarean sections done 9 years and 7 years ago. The patient had a history of self-medicating for termination of pregnancy at 7 weeks of amenorrhea. She had heavy vaginal bleeding for which she had consulted another doctor, where she had been diagnosed with an incomplete abortion. The patient was prescribed the tablet Misoprostol initially and when it was not effective, dilation and curettage (D\&C) had been done. Postevacuation, ultrasonography (USG) still showed retained products of conception (RPOC), for which she was again prescribed misoprostol, which she did not take. She was asymptomatic for 2 weeks after which she had another bout of heavy vaginal bleeding, at which time she presented to our hospital. On examination, she was pale, with stable vitals. Pelvic examination revealed a normalsized uterus and no active bleeding. USG examination showed 3.2 $\times 3.8 \times 3.9 \mathrm{~cm} \mathrm{RPOC}$ at the scar site, and the scar itself appeared thinned out. Suspecting scar pregnancy, magnetic resonance imaging (MRI) of the pelvis was done, which showed cesarean scar
1-3,5S Nijalingappa Medical College and Hanagal Shree Kumareshwar Hospital, OBG, Bagalkot, Karnataka, India

${ }^{4}$ Department of Radiology, S Nijalingappa Medical College and Hanagal Shree Kumareshwar Hospital, OBG, Bagalkot, Karnataka, India Corresponding Author: Veena Hadi, S Nijalingappa Medical College and Hanagal Shree Kumareshwar Hospital, OBG, Bagalkot, Karnataka, India, Phone: +91 7760329621, e-mail: veenahadi@gmail.com

How to cite this article: Rao S, HadiV, Mallapur A, et al. Myriad Obstetric Complications in Patients with Scarred Uterus: A Case Series. J South Asian Feder Obst Gynae 2020;12(6):438-442.

Source of support: Nil

Conflict of interest: None

RPOC of aforementioned dimensions with thinning of anterior myometrium (maximum thickness $4 \mathrm{~mm}$ ) and no extension beyond serosa (Fig. 1). Options of methotrexate injection, scar excision with uterine repair, and hysterectomy were discussed with the patient and relatives. The patient was electively posted for laparotomy with scar excision and resuturing. Intraoperatively, the scar appeared to be very thin (Fig. 2) and the products came out of the scar as soon as the uterovesical fold of peritoneum was separated (Fig. 3). The products of conception (confirmed by histopathology later)

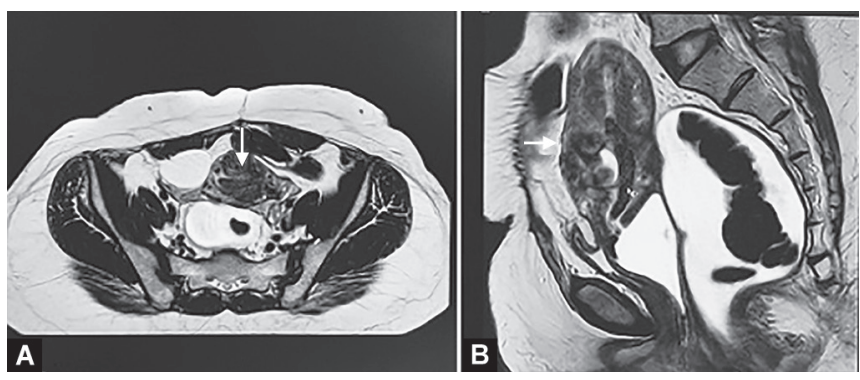

Figs $1 \mathrm{~A}$ and B: MR images of case 1 showing cesarean scar RPOC: axial and sagittal. T2 W images show $T 2$ hypointense placental tissue in the anterior myometrium $(\mathrm{A})$ at the scar region with thin intact serosa $(\mathrm{B})$

( ) Jaypee Brothers Medical Publishers. 2020 Open Access This article is distributed under the terms of the Creative Commons Attribution 4.0 International License (https://creativecommons.org/licenses/by-nc/4.0/), which permits unrestricted use, distribution, and non-commercial reproduction in any medium, provided you give appropriate credit to the original author(s) and the source, provide a link to the Creative Commons license, and indicate if changes were made. The Creative Commons Public Domain Dedication waiver (http://creativecommons.org/publicdomain/zero/1.0/) applies to the data made available in this article, unless otherwise stated. 


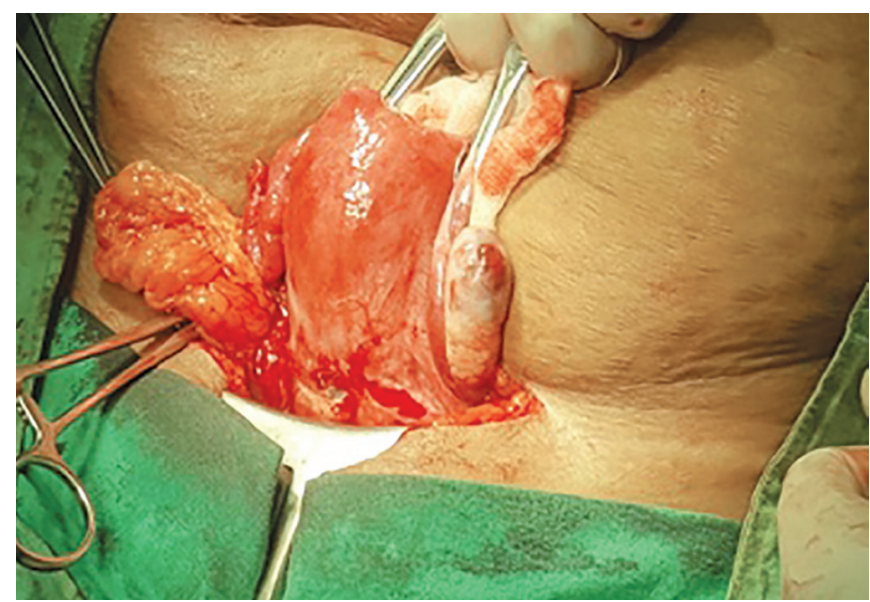

Fig. 2: Thinned out scar noted on laparotomy

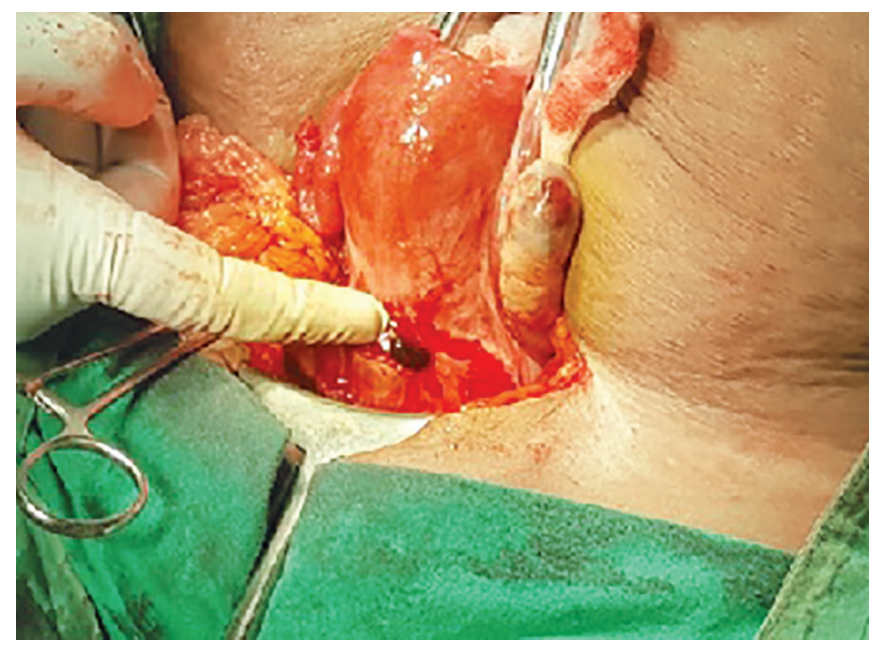

Fig. 3: Products of conception were seen as soon as uterovesical fold of peritoneum was separated
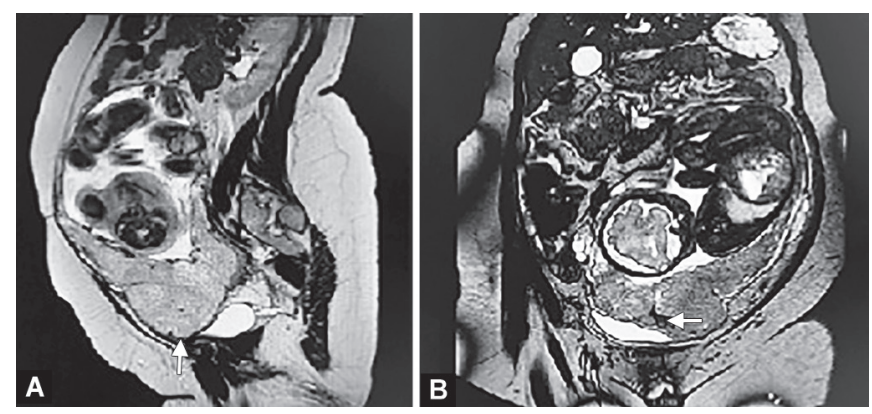

Figs 4A and B: MR images of case 2 with placenta accreta:T2 sagittal (A) and coronal (B) images show complete placenta previa with heterogeneous signals with lumpy contour, round edges of the placental tissue (upward arrow), and $\mathrm{T} 2$ hypointense intraplacental band (side arrow)

were removed, followed by scar excision and uterine repair. The patient withstood the procedure well and was discharged on the 7th postoperative day.

\section{Case 2: Central Placenta Previa with Placenta Accreta}

Twenty-six-year-old Mrs. B with an obstetric index of G3P2L2 with 31 weeks of gestation presented with no complaints, with an ultrasound report showing a single live intrauterine gestation with central placenta previa with thinning of anterior myometrium with suspicion of placenta accreta. She was admitted for further evaluation and safe confinement. MRI abdomen and pelvis showed central placenta previa with a focal uterine bulge on the left side of the uterus approximately $2 \mathrm{~cm}$ above the internal os, with features suggestive of placenta accreta. However, no placental tissue was detected outside the serosal layer (Fig. 4). The patient stayed in the hospital for observation. The patient remained asymptomatic and was taken up for elective cesarean section with rescue peripartum hysterectomy if needed at 36 weeks of gestation. Intraoperatively, a classical incision was put on the uterus but the placenta was encountered along the entire length of the incision. Hence, placenta was cut through for the extraction of the baby. A healthy live female baby weighing $2.6 \mathrm{~kg}$ was extracted. The patient had postpartum hemorrhage which was controlled by multiple compression sutures taken through and through the anterior and posterior walls of the uterus. The part of the placenta which was cut was removed and the rest of the placenta was left in situ. Intraoperatively, two units of packed red blood cells (pRBCs) and two units of fresh frozen plasma (FFP) were transfused and one unit of pRBC was transfused postoperatively. Methotrexate injection $50 \mathrm{mg}$ was alternated with Leucovorin injection $5 \mathrm{mg}$ for a total of five doses each, starting on the 6th postoperative day. Day 17 USG showed placental tissue of dimensions $10 \times 11 \times 8 \mathrm{~cm}$ with no vascularity. The patient was discharged and was followed up for any signs of sepsis or bleeding. The patient had decreasing size of placental tissue at sequential follow-up visits, with complete resolution noted at 3 months postpartum.

\section{Case 3: Placenta Previa Increta Masking Ruptured Uterus}

Twenty-six-year-old Mrs. C presented to the labor room on an emergency basis with a history of 7 months of amenorrhea and complaints of vaginal bleeding for 2 hours. Her obstetric score was G3P2L2 with 26 weeks of gestation with previous two cesarean sections done 9 years and 7 years back. The first cesarean section was done in view of oligohydramnios and the second one was done for placenta previa. On examination, the patient's vitals were stable, abdominal examination showed a uterus of 26 weeks size, and speculum examination showed no active bleeding. The patient had a USG report at 20 weeks gestation showing placenta covering the internal os and loss of the retroplacental hypoechoic zone, suggestive of placenta accreta. The patient was admitted for observation and evaluation. MRI pelvis done showed central placenta previa with features suggestive of placenta increta (Fig. 5). Admission was continued for surveillance of maternal and fetal condition. At about 31 weeks of gestation, the patient complained of epigastric pain which subsided partially with antacids. But 4 hours later, upper abdominal pain increased and the patient had two episodes of vomiting. She also manifested increasing tachycardia and fall of blood pressure (BP). Emergency laparotomy was done and intraoperatively it was found that there was a midline vertical rupture (about $4 \mathrm{~cm}$ length) in the upper segment of the uterus with placental tissue protruding through the rent (Fig. 6). This rent was extended downward and after cutting through the placenta, a preterm baby weighing $1.7 \mathrm{~kg}$ was extracted. Decision was made to proceed with obstetric hysterectomy (Fig. 7). Intraoperative blood loss was around $2000 \mathrm{~mL}$. A total of three units of $\mathrm{pRBC}$ and two units of FFP were transfused. She had some derangements of the 


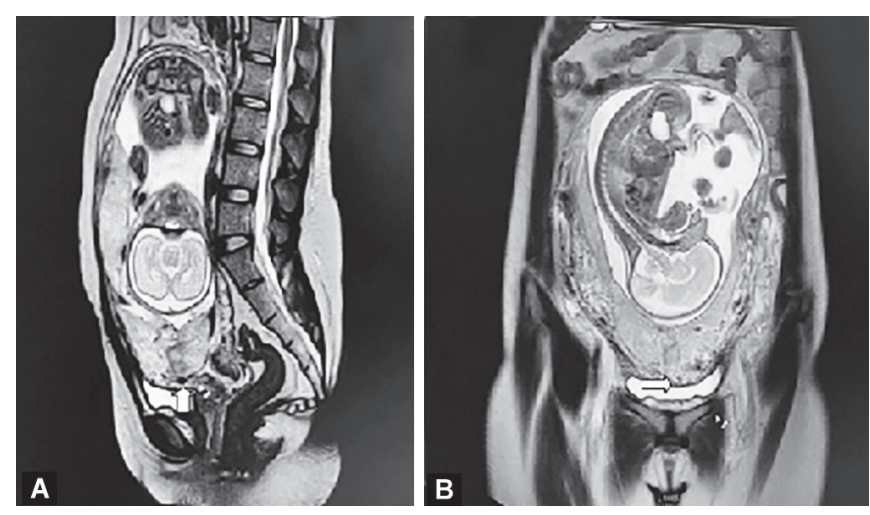

Figs $5 A$ and B: MR images of case 3 showing placenta increta:T2 sagittal and coronal images show complete placental previa with prominent T2 flow void vascular channels (A) and loss of interface between the decidua basalis and uterine myometrium (B)

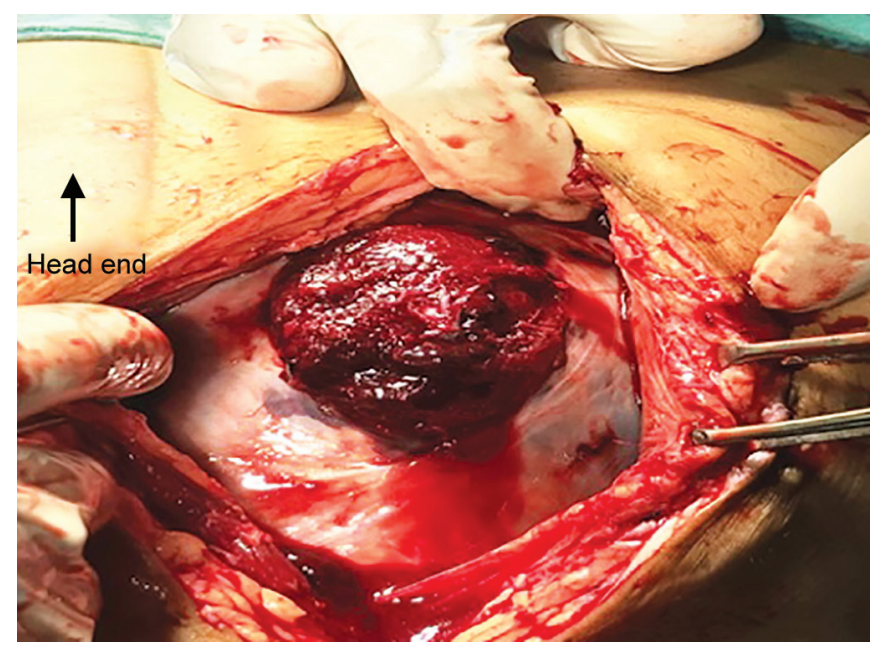

Fig. 6: Placenta protruding through the rent in the upper segment of the uterus

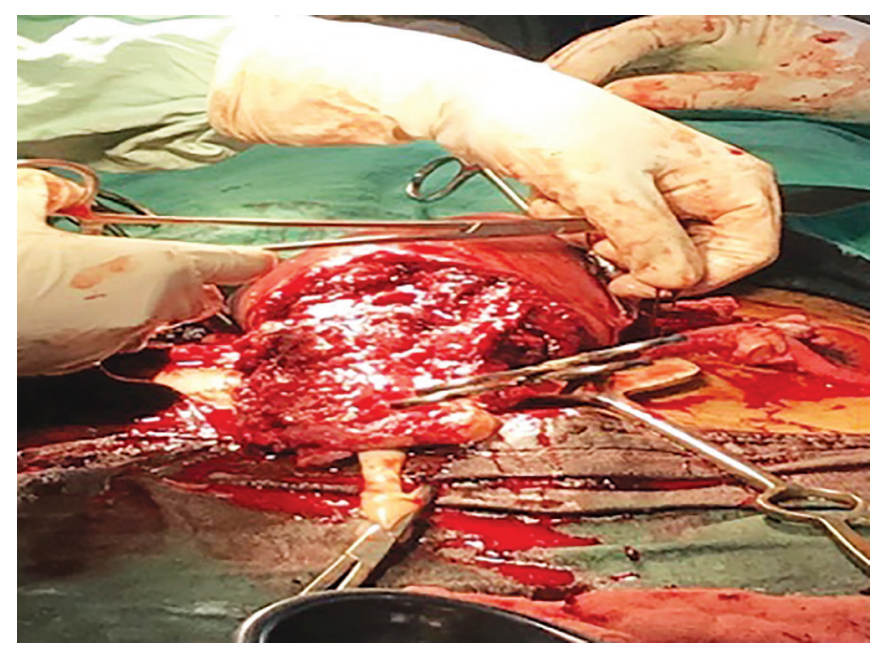

Fig. 7: Completely adherent placenta

liver and renal function following surgery, which improved with multidisciplinary treatment. Both the patient and the baby were discharged in stable condition on the 12th postoperative day.

\section{Case 4: Septic Shock with Adherent Placenta}

Twenty-eight-year-old Mrs. D presented to the emergency room with history of 4 months of amenorrhea with complaints of fever with chills since 2 days and leaking per vaginum since 1 day. She was G4P2L1A1 with previous two cesarean sections done 8 years and 6 years back and one abortion in which $D \& C$ had been done 3 years back. On examination, the patient was conscious, oriented but had cold peripheries with a pulse rate (PR) of 130 beats/ minute, BP of $74 / 36 \mathrm{~mm} \mathrm{Hg}$, and respiratory rate (RR) of 28 cycles/ minute. Abdominal examination revealed a tender uterus of 14-16 weeks size. Speculum examination revealed foul-smelling vaginal discharge. On digital vaginal examination, it was found that the cervix was soft, tubular, $2.5 \mathrm{~cm}$ long, and $1 \mathrm{~cm}$ dilated with absent membranes. Initial measures of stabilization were undertaken, the patient was started on noradrenaline drip, and higher antibiotics were initiated. USG showed an intrauterine dead fetus of 12 weeks with absent liquor, with no evidence of pelvic collection. A diagnosis of septic abortion with septic shock was made. Misoprostol 200 $\mu \mathrm{g}$ was administered vaginally for termination of pregnancy. After about 6 hours, a macerated abortus was expelled but even 45 minutes later, there were no signs of placental expulsion. By this time the patient's general condition deteriorated, with temperature $104^{\circ} \mathrm{F}, \mathrm{PR} 148$ beats/minute, BP 140/80 $\mathrm{mm} \mathrm{Hg}$ (on noradrenaline infusion) and RR 45 cycles/minute. The decision for evacuation under anesthesia was taken. Considering her overall status, she was electively intubated. During the procedure, the placenta was found firmly adherent to the anterior uterine wall and was removed piecemeal. A moderate amount of hemorrhage occurred, which was managed medically. The patient was shifted to the intensive care unit for further monitoring. With higher antibiotics and ventilatory support, the patient recovered gradually and was extubated on the 5th postoperative day. USG on the 6th postoperative day showed RPOC of dimensions $2.8 \times 1.7 \times 1.9 \mathrm{~cm}$ without vascularity. The patient was managed conservatively as she was asymptomatic and was discharged in stable condition on 10th postoperative day. At follow-up 2 weeks later, a repeat USG was done, which showed complete resolution of the remaining placental tissue.

\section{Case 5: Rare Case of Vesicocutaneous Fistula after Third Repeat Cesarean Section}

Thirty-three-year-old Mrs. E was referred on day 5 postcesarean section as she had developed bleeding from the suture site. Her obstetric score was P3L2 with previous three cesareans. The last cesarean section was done for abruption placenta with intrauterine fetal demise at 28 weeks of gestation. The patient was a known case of severe preeclampsia and was on antihypertensives. The referral card mentioned that as dense adhesions were encountered between the anterior abdominal wall and the uterus, surgery had been done through limited access and that the peritoneal cavity could not be entered. Retroplacental clots were present, and also, the placenta was found adherent in the lower uterine segment. She had received three units of pRBC and two units of FFP during the surgery. She was referred on day 5 following surgery due to bleeding from the abdominal wound. In our hospital, on examination, the patient's general condition was stable. There was continuous seepage of altered blood from the wound site. The rest of the abdominal and pelvic findings were not clearly made out. USG showed collection within the uterus and also between the uterus and the anterior abdominal wall. The patient was taken up for exploratory laparotomy. Intraoperatively, a collection of around 300 
$\mathrm{mL}$ of foul-smelling altered blood was found, which was evacuated. The uterine suture site had sloughed off completely. Uterine edges were freshened, and resuturing was done. Dense adhesions again prevented entry into the peritoneal cavity. A drain was kept below and above the rectus sheath. The immediate postoperative period was uneventful with minimal drain output, which was removed on the 4th postoperative day. On the 9th day, she developed watery discharge from the entire length of the wound, which had gaped partially. Analysis of the discharge confirmed it to be urine. The bladder was catheterized with Foley's catheter, after which the discharge subsided. Intravenous urography did not reveal any abnormality. It was decided to try conservative management of the vesicocutaneous fistula with continuous bladder drainage and tablet Tolterodine. On postoperative day 16, the abdominal wound appeared to be healing and the patient was discharged with Foley's catheter in situ. At the time of this report (6 weeks since surgery), the patient is still on follow-up with the catheter in situ and no obvious leak through the wound.

\section{Discussion}

In the era of rising cesarean sections, management of cases with previous one or more cesarean sections has become the norm rather than the exception. There are various guidelines available for the management of cases with previous cesarean in labor. However, patients with a scarred uterus may present at any time during pregnancy with a host of complications. Treating such complications lead to a lot of diagnostic and management dilemmas as there is no definite prescribed protocol and individualized management strategies need to be adopted.

Scar pregnancies have found a new place in the classification of ectopic pregnancies, as an iatrogenic location for pregnancy implantation. ${ }^{2}$ In case 1 , scar pregnancy was not diagnosed as the patient directly presented after taking pills for abortion. Various procedures have been described for the management of scar pregnancy. Conservative methods described include (1) evacuation of the pregnancy and repair of the uterine defect by laparoscopy or laparotomy ${ }^{3,4} ;(2)$ visually guided suction curettage with or without methotrexate; (3) administration of methotrexate locally or intramuscularly. ${ }^{5}$ Combinations of the described methods have also been used with success, such as systemic methotrexate followed by D\&C. ${ }^{6}$ In all such cases, uterine artery embolization can be used to decrease the chance of hemorrhage. ${ }^{7}$ Hysterectomy by laparotomy or laparoscopy is an acceptable choice in the patients who desire sterilization. ${ }^{2}$ Godin et al. reported a case of scar pregnancy which was managed by injecting potassium chloride into the fetus and methotrexate into the gestational sac through the vaginal route. ${ }^{8}$ But dehiscence of the scar was noted after 16 weeks. In our case, we encountered the patient when only RPOC was present at the scar. But as the myometrial thickness was noted to be very thin ( $4 \mathrm{~mm}$ ), the option of hysteroscopic removal was discarded as it was doubtful whether the scar would withstand hydrodistension. The option of methotrexate injection was not considered as there was no vascularity in the RPOC. Hence, scar excision and resuturing of the uterine edges as described by Comert et al. as in method 1 above were considered to be most suitable.

Placenta accrete spectrum (PAS) is encountered more frequently in current obstetrics due to increasing rates of cesarean deliveries. PAS including accrete, increta, and percreta is becoming a common indication for obstetric hysterectomy. ${ }^{9}$ An alternate well-accepted approach is the conservative method, in which the placenta is left in situ, followed by systemic methotrexate administration. The conservative management is associated with chances of secondary postpartum hemorrhage, delayed secondary hysterectomy, and sepsis. So, in managing such cases we need to consider these factors, along with the patients' parity and compliance to follow-up. This conservative approach was adopted with success in our second case, although it took 3 months for the complete resolution of the placenta.

Case 3 was an unusual presentation of placenta increta. Even though the patient presented with antepartum hemorrhage initially, she did not have any vaginal bleeding subsequently. Upper abdominal pain was the predominant symptom of the ruptured uterus which was not diagnosed initially. On retrospective analysis, the rupture in the upper segment when the patient was not in labor could be due to prior classical or inverted $T$ incision as the previous section was done for placenta previa. This could not be ascertained due to the nonavailability of a prior discharge card. Not having access to the previous discharge summary when managing subsequent pregnancy is a common occurrence hence it is very important to educate the patient or relatives about any issues or complications which are likely to influence future obstetric performance.

In the fourth case, the decision of termination with Misoprostol was taken to avoid the risk of peritonitis due to laparotomy which would have worsened the patient's condition. Even though Misoprostol is contraindicated with previous cesarean deliveries, there are reports of using it even in a case of five previous sections. ${ }^{10}$ In our case also it was successful in the expulsion of the fetus but due to adherent placenta, we had to go for uterine evacuation under anesthesia. Hence, the possibility of adherent placenta should be kept in mind in all cases with previous scar on the uterus.

Case 5 was a very rare case of vesicocutaneous fistula, occurring as a result of complication of repeat cesarean sections. The case was further complicated by sepsis which had caused sloughing of the uterine edges. It is likely that attempts to close the uterus and achieve hemostasis might have resulted in unnoticed bladder trauma, which resulted in vesicocutaneous fistula. Most cases of vesicocutaneous fistulae reported in the literature were secondary to trauma to the pelvis ${ }^{11}$ or radiation induced. ${ }^{12}$ Toufique et al. have reported one case after third repeat cesarean section which ended in obstetric hysterectomy. ${ }^{13}$ The authors have reported success with conservative management of the vesicocutaneous fistula, although other authors have described various surgical repair techniques. ${ }^{14-16}$

All the five patients in this series had two previous cesarean sections. It is to be noted that of the five patients, three did not want a child in the current reported pregnancy. Contraception, in the form of either concurrent sterilization or transcesarean copper-T insertion with sterilization at a later date, should be considered in every repeat cesarean section, to limit the occurrence and hence complications of unwanted postcesarean pregnancies. Even while performing the primary cesarean section, copper-T insertion should be advocated for spacing, as majority of our women do not have the knowledge, will, or autonomy to seek contraceptive advice. $^{17}$

\section{Conclusion}

Management of cases of previous cesarean sections can be challenging as there is a steady increase in a variety of placental and scar complications. Identifying atypical presentations at the earliest and referring such patients to tertiary care centers 
are important. Good blood bank facilities and management by a multidisciplinary team involving obstetricians, radiologists, urologists, and intensivists are vital for the best possible care of the patient, to minimize maternal morbidity and mortality.

\section{References}

1. Practice Bulletin No. 184: Vaginal birth after cesarean delivery. Obstet Gynaecol 2017 Nov;130(5):217-233. DOI: 10.1097/ AOG.0000000000002398.

2. Cunningham FG, Levono KJ, Bloom SL, et al., editors. Williams obstetrics. 25th ed. New York, NY: McGraw-Hill; 2018. p. 909-950.

3. Cömert EH, Sal H, Ekici YS, et al. Cesarean scar pregnancy: a case report. Turkiye Klinikleri Jinekoloji Obstetrik 2018;26(1):37-39. DOI: 10.5336/gynobstet.2016-53978.

4. Patel MA. Scar ectopic pregnancy. J Med Biol Eng 2015;65(6):372-375. DOI: 10.1007/s13224-015-0817-3.

5. Fylstra DL, Pound-Chang T, Miller MG, et al. Ectopic pregnancy within a cesarean delivery scar: a case report. Am J Obstet Gynecol 2002;187(2):302-304. DOI: 10.1067/mob.2002.125998.

6. Graesslin O, Dedecker F, Quereux C, et al. Conservative treatment of ectopic pregnancy in a cesarean scar. Obstet Gynecol 2005;105(4):869871. DOI: 10.1097/01.AOG.0000141569.75297.44.

7. Badawy SZ, Etman A, Singh M, et al. Uterine artery embolization: the role in obstetrics and gynecology. Clin Imaging 2001;25:288-295. DOI: 10.1016/s0899-7071(01)00307-2.

8. Godin PA, Bassil S, Donnez J. An ectopic pregnancy developing in a previous Cesarean section scar. Fertil Steril 1997;67(2):398-400. DOI: 10.1016/S0015-0282(97)81930-9.
9. Berhan $Y$, Urgie T. A literature review of placenta accreta spectrum disorder: the place of expectant management in Ethiopian Setup. Ethiop J Health Sci 2020;30(2):277-292. DOI: 10.4314/ejhs. v30i2.16.

10. AISaad D, Al-Obaidly S, Abdulrouf $\mathrm{P}$, et al. Misoprostol for miscarriage management in a woman with previous five cesarean deliveries: a case report and literature review. Ther Clin Risk Manag 2017 May 9;13:625-627. DOI: 10.2147/TCRM.S132294.

11. Kotkin L, Koch MO. Morbidity associated with non operative management of extraperitoneal bladder injuries. J Trauma 1995;38(6):895-898. DOI: 10.1097/00005373-199506000-00012.

12. Lau KO, Cheng C. A case report: delayed vesicocutaneous fistula after radiation therapy for advanced vulvar cancer. Ann Acad Med Singapor 1998;27(5):705-706.

13. Toufique H, Merani AJ. Vesicocutaneous fistula. J Pak Med Assoc 2011 Sep;61(9):918-919

14. Freeman JJ, Storto DLP, Berry-Cabán CS. Repair of a vesicocutaneous fistula using negative-pressure wound therapy and urinary diversion via a nephrostomy tube. J Wound Ostomy Continence Nurs 2013;40(5):536-538. DOI: 10.1097/WON.0b013e3182a46549.

15. Kim SW, Lee JN, Kim HT, et al. Management of vesicocutaneous fistula presenting 13 years after radiotherapy for cervical cancer. Turk J Urol 2018; 44(2):185-188. DOI: 10.5152/tud.2017.35929.

16. Hennessey DB, Bolton $\mathrm{E}$, Thomas AZ, et al. Vesicocutaneous fistula following adjuvant radiotherapy for prostate cancer. BMJ Case Rep. 2013;2013. PII: bcr2013008986. DOI: 10.1136/bcr-2013-008986.

17. Sinha A. Women's status, autonomy, and contraceptive behaviour in India: a re-examination of Indian DHS data. APA Conf Paper 2015 DOI: $10.13140 / 2.1 .4649 .5522$. 